\title{
Genetic structure of the abd-A gene of Drosophila
}

\author{
ANA BUSTURIA*, JORDI CASANOVA**, ERNESTO SÁNCHEZ-HERRERO***, ROSA GONZÁLEZ \\ and GINÉS MORATA
}

Centro de Biología Molecular, Universidad Autonoma de Madrid, Canto Blanco, Madrid 28049, Spain.

Authors present addresses: *Zoologisches Institut der Universitat Zurich, Wintherthurestrasse 190, 8057 Zurich, Switzerland. **Neurobiology and Behaviour Center, Columbia University, 722 West 168th St., New York NY 10032.

*** Genetics Department, University of Cambridge, Downing Street, Cambridge CB2 3EH

\begin{abstract}
Summary
We report the embryonic and adult phenotypes of a number of mutations of the $a b d-A$ gene of the bithorax complex. Some of them result in loss of $a b d-A$ function in the whole $a b d-A$ domain and are usually lethal. These probably eliminate or inactivate $a b d-A$ protein products. Other mutations affect only part of the $a b d-A$ domain. These are viable, appear to map outside the $a b d-A$ transcription unit, and presumably alter the normal spatial regulation of $a b d-A$ products.
\end{abstract}

We propose a model of $a b d-A$ structure based on a protein-coding region and two cis-regulatory regions. Regulatory region $1,3^{\prime}$ to the transcription unit, contains positive and negative regulatory elements. Regulatory region $2,5^{\prime}$ to the transcription unit, establishes the correct level of $a b d-A$ activity in the abdominal metameres.

Key words: Drosophila, homeotic genes, bithorax complex.

\section{Introduction}

The bithorax complex (BX-C) specifies the identity of the portion of the body of Drosophila between and including parasegments 5 and 13 (Lewis, 1978; SánchezHerrero et al. 1985). The BX-C consists of three genes (Sánchez-Herrero et al. 1985; Tiong et al. 1985) termed Ultrabithorax $(U b x)$, abdominal-A $(a b d-A)$ and $\mathrm{Ab}-$ dominal-B $(A b d-B)$ each one containing a homeobox (McGinnis et al. 1984; Scott and Wiener, 1984; Regulski et al. 1985). Of these, $a b d-A$ is responsible for the specific identity of parasegments 7 to 9 and is also necessary for the correct development of parasegments 10 to 13 . Like the rest of the BX-C, $a b d-A$ has been cloned and there is a preliminary molecular characterization of its structure (Karch et al. 1985; Regulski et al. 1985; Rowe and Akam, 1988). A number of mutations of $a b d-A$ have been reported (Sánchez-Herrero $e t a l$. 1985; Tiong et al. 1985; Karch et al. 1985) but the genetic and phenotypic description of many of these mutations is still incomplete. There is not a genetic dissection of $a b d-A$ comparable to those of $U b x$ and $A b d-B$ (Casanova et al. 1985a; Peifer and Bender, 1986; Casanova et al. 1986).

In this paper, we describe 19 mutations of $a b d-A$, some of which have been defined molecularly by Karch et al. 1985. Our principal aim is to understand the functional components of $a b d-A$. We find that some $a b d-A$ mutations eliminate or severely reduce $a b d-A$ functions and map at or close to the homeobox- containing unit. Other $a b d-A$ mutations map outside the transcription unit and alter the local distribution of $a b d-A$ products. The latter define regulatory regions of the gene.

\section{Materials and methods}

\section{abd-A mutations used and their names}

The majority of the abd-A mutations used in this work (Table 1) have already been reported, but not described, in papers by Lewis, 1978; Kuhn et al. 1981; Sánchez-Herrero et al. 1985; Tiong et al. 1985 and Karch et al. 1985. Appropriate references are given in the main text. Some mutations have been induced in our laboratory while others come from other sources, especially from Welcome Bender and Francois Karch. In naming the mutations in this paper, we have followed the suggestion of Duncan (1987) and have distinguished between mutations affecting the whole domain, labelled $a b d-A$, and those only affecting part of the domain, which are named infraabdominal ( $i a b$ ) followed by a number indicating the abdominal segment where they have the most prominent effect. This nomenclature, while sensible, is not devoid of problems; mutations of the second category are often difficult to classify because they affect similarly more than one metamere. Moreover, many of them exhibit dominant gain-of-function as well as recessive loss-of-function phenotypes. In renaming some of the mutants, we have considered only the recessive phenotypes.

We also describe here some new mutations: $a b d-A^{\mathrm{MX} 3}$ and $i a b-4^{\mathrm{MX} 4}$, which have been X-ray-induced in our laboratory, 
and $i a b-2^{\mathrm{M} 1}$, that appeared spontaneously in a $C y / P m$; $S b / T M 2$ stock.

Except in the cases described below, all these mutations complement those of $U b x$ and $A b d-B$, hence only the $a b d-A$ gene is altered. The exceptions are $a b d-A^{\mathrm{C} 51}$ which contains a $b x$ mutation, $i a b-2^{\mathrm{Uab} 1}$ which has a breakpoint in $b x d$ and another in the $r$ region of $A b d-B$ (Karch et al. 1985; Casanova et al. 1986) and finally iab-4,5 $5^{\mathrm{DB}}$ which is associated with a deletion removing parts of both $a b d-A$ and $A b d-B$. The presence of these mutations does not affect the $a b d-A$ phenotypes reported in this paper as the tester chromosomes we have used (see Results) have normal doses of $U b x$ and $A b d-B$.

In many instances in which it was necessary to discriminate the dominant from the recessive phenotype of the $a b d-A$ mutations, we used the $T(1 ; 3) P 115$. In this rearrangement (see Casanova et al. $1985 b$ for description), a chromosomal portion, ( $D p P 115)$ carrying a normal set of $\mathrm{BX}-\mathrm{C}$ genes has been translocated to the first chromosome. It can be segregated to produce flies with supernumerary copies of the BXC.

\section{Measure of the homeotic transformations}

The assessment of homeosis in the abdominal segments is difficult because they have similar patterns. We find it, for example, very hard to distinguish the third (A3) from the fourth (A4) abdominal segment, therefore we may have overlooked transformations of one into the other. The segment $\mathrm{A} 1$ is clearly different from the rest because it contains in the dorsal side (tergite) bristles that are finer and shorter than those of the other segments. It also contains a characteristic cuticular arch. The posterior compartment of A1 (Kornberg, 1981) is the only posterior abdominal compartment that can be identified morphologically in the adult. On the ventral side (sternite), the A1 segment has no bristles whereas the other segments have between 15 and 20 . Thus any transformation of $\mathrm{Al}$ into a more posterior segment or vice versa can readily be distinguished.

Transformations involving segments $\mathrm{A} 2-\mathrm{A} 4$ are the most difficult to see because their tergites are very similar. Ventrally the sternite of $\mathrm{A} 2$ has a distinctive bristle pattern and contains a Wheeler's organ. Also the pair of sensillae that appear in the anterior region are further apart in A2 than in other sternites. The A5 and A6 segments can be distinguished in male flies on the basis of the pigmentation of the tergite and the number of the bristles of the sternite of A6.

\section{Cuticle preparations}

Embryos were prepared for microscopic inspection following the standard method of Van der Meer (1977). Adult cuticle was digested with hot $\mathrm{KOH}$, dehydrated with propanol and mounted in Euparal.

\section{$\beta$-gal staining of the adult cuticle}

Abdomens were cut from freshly eclosed flies fixed in $0.1 \%$ glutaraldehyde (Fluka) and washed with PBS. They were then transferred to $\mathrm{X}$-gal solution $(0.2 \%$ 5-bromo-4chloro-3indoly- $\beta$-D-galactopyranoside in $10 \mathrm{~mm}$-sodium pyrophosphate, $0.5 \mathrm{M}-\mathrm{NaCl}, 1.0 \mathrm{~mm}-\mathrm{MgCl}_{2}, 5 \mathrm{~mm}$-potassium ferricyanide, $5 \mathrm{~mm}$-potassium ferrocyanide) and stained for several hours at $37^{\circ} \mathrm{C}$. Stained abdomens were then dehydrated through a series of alcohols and mounted in Canada Balsam.

\section{Results}

(1) Classification of the abd-A mutations used

All the mutations at the $a b d-A$ locus used in this work are listed in Table 1 . They have been characterized with reference to the combination DpPIO DflOg (Morata et al. 1983), which we consider as a complete lack of $a b d-A$ function. This is lethal and gives a very strong $a b d-A$ phenotype. In this genotype, most of $a b d-A$ DNA, from +33-36 to +86-93 (Karch et al. 1985), is deleted. Thus no $a b d-A$ protein product can originate from this combination. It complements all $A b d-B$ alleles, so the $A b d-B$ gene appears to be intact. It also contains a normal $U b x$ gene in the $D p P l O$ fragment.

We have divided the mutations in two groups: (1) those that have loss-of-function phenotypes similar to that of DpP10 Df709 (heretofore referred to as Df), which affect the entire $a b d-A$ domain and (2) those whose phenotypes affect only part of the $a b d-A$ domain and/or which are associated with gain of function of $a b d-A$ outside the normal domain. Those of the first group will probably eliminate or inactivate executive $a b d-A$ gene products, whereas the second group will include mutations altering regulatory elements of the gene.

(2) Mutations eliminating or reducing abd-A function in the entire abd-A domain

Except those involving $a b d-A^{\mathrm{M} 3}$, all the homozygotic or trans-heterozygotic combinations of these mutations were zygotic lethal. We have examined the hemizygous embryonic phenotype of all them. Those of $D f$ and $a b d-A^{\mathrm{P} 10}$ (formerly $T(2 ; 3 P 10)$ ) have already been described (Morata et al. 1983).

Except in the cases of $a b d-A^{\mathrm{P} 10}, a b d-A^{\mathrm{C} 26}$ and $a b d-A^{\mathrm{M} 3}$, the embryonic phenotypes were very similar and like that of $D f$; abdominal parasegments 7-9 are completely transformed into parasegment 6 and parasegments $10-13$ also resemble parasegment 6 but the transformation is not complete (Sánchez-Herrero et al. 1985). Of the exceptions, $a b d-A^{\mathrm{P} 10}$ and $a b d-A^{\mathrm{C} 26}$ show

Table 1. abd-A mutations

\begin{tabular}{|c|c|c|c|}
\hline $\begin{array}{l}\text { Affecting the } \\
\text { entire domain }\end{array}$ & Position & $\begin{array}{l}\text { Affecting part } \\
\text { of the domain }\end{array}$ & Position \\
\hline 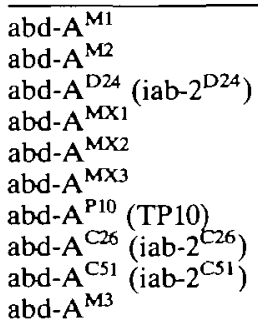 & $\begin{array}{l}+42 \\
\text { nd } \\
\text { nd } \\
+56 \\
+57 \\
\text { nd } \\
+33 \\
+35 \\
+43 \\
\text { nd }\end{array}$ & 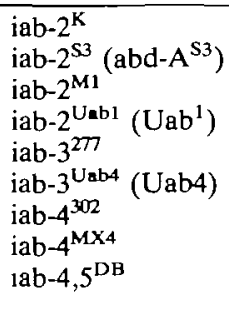 & $\begin{array}{l}+27,5 \\
+26 \\
\quad \text { nd } \\
-14^{\star},+168^{*} \\
+64 \\
+63 \\
+86 \\
\quad \text { nd } \\
+(85-113)\end{array}$ \\
\hline
\end{tabular}

nd, not determined.

* These breaks are outside the $a b d-A$ gene.

Position of the mutations are given in kilobases as in Karch et al. 1985 and Weiffenbach and Bender (personal communication). In the cases we are changing the name of the mutation, the old name is in parenthesis. 
less extreme phenotypes, indicating that $a b d-A$ is not wholly inactive. This is supported by the finding that epidermal cell clones hemizygous for $a b d-A^{\mathrm{P} 10}$ show partial adult transformations whereas in the cases of $D f$ or $a b d-A^{\mathrm{M} 1}$ adult transformations are more extreme (Morata et al. 1983; Sánchez-Herrero et al. 1985). Nevertheless, embryos hemizygous for either $a b d-A^{\text {P10 }}$ or $a b d-A^{C 26}$ have all abdominal segments (except $\mathrm{A} 1$ ) affected and this comprehensive phenotype is probably responsible for the lethality.

The hemizygous viable mutation $a b d-A^{\mathrm{M} 3}$ shows a very weak embryonic phenotype; it can only be detected as some reduction in the width of the denticle belt of A2-A4 segments. This may be an indication of transformation towards A1.

The adult phenotype of $a b d-A^{\mathrm{M} 3}$ in combinations with the other mutations of this class is characterized by a general effect on segments $\mathrm{A} 2-\mathrm{A} 6$; the number of bristles in the sternites of all these segments is reduced, although the effect on $\mathrm{A} 2$ is more marked in the tergites where we observe the presence of some fine, short bristles and often a lateral cuticular arch. These are characteristic pattern elements of $\mathrm{A} 1$. In addition, we have observed in the naked territory between tergites some tubular structures which resemble the posterior compartment of T3p. By en- $\beta$ gal staining (Busturia and Morata, 1988), we have shown that these structures belong to the posterior compartment. These results suggest that in $a b d-A^{\mathrm{M} 3} / D f$ flies there is a weak transformation of parasegments 7,8 and 9 towards parasegment 6 (T3p-Ala).

The combinations of $a b d-A^{\mathrm{M} 3}$ with $a b d-A^{\mathrm{P} 10}$ $a b d-A^{\mathrm{C} 26}, a b d-A^{\mathrm{MX} 1}, a b d-A^{\mathrm{MX} 2}$ and $a b d-A^{\mathrm{MX} 3}$ present a weaker phenotype than the rest. This was expected for $a b d-A^{\mathrm{P} 10}$ and $a b d-A^{\mathrm{C} 26}$ but not for $a b d-A^{\mathrm{MX} 1}$ $a b d-A^{\mathrm{MX} 2}$ and $a b d-A^{\mathrm{MX} 3}$ which exhibit strong embryonic phenotypes. It suggests that the latter mutations may not completely inactivate $a b d-A$.

Some of these mutations have been localized on the DNA by Karch et al. 1985 (see also Duncan, 1987) as shown in Table 1 . They define a DNA interval from $+35 \mathrm{~kb}$ to $+57 \mathrm{~kb}$, which includes the homeobox-containing transcription unit (Karch et al. 1985; Rowe and Akam, 1988). Therefore, these mutations eliminate or inactivate $a b d-A$ protein products. The cases of $a b d-A^{\mathrm{P} 10}, a b d-A^{\mathrm{C} 26}$ and $a b d-A^{\mathrm{MX} 2}$ do not conform to this rule, for their moderate phenotypes indicate that there is $a b d-A$ product left. However, the first two mutations map around $+35 \mathrm{~kb}$, very close to the $3^{\prime}$ end of the transcription unit, whereas $a b d-A^{\mathrm{MX} 2}$ is located around $+57 \mathrm{~kb}$, very close to the $5^{\prime}$ end. In these cases, we suspect the lesions may lie just outside the coding unit or, if inside, there still is some functional protein produced.

\section{(3) Mutations affecting part of the abd-A domain}

As we indicated above these mutations are likely to alter regulatory elements of the gene. We describe them in the order they are listed in Table 1 according to the adult abdominal segment where they have the most prominent effect, starting from $\mathrm{A} 1-\mathrm{A} 2$.

\section{Infraabdominal $-2^{\mathrm{K}}\left(\mathrm{iab}-2^{\mathrm{K}}\right)$}

It is caused by a gypsy transposon inserted at $+27.5 \mathrm{~kb}$ (Karch et al. 1985). The adult phenotype of $i a b-2^{\mathrm{K}}$ was first described by Kuhn et al. 1981 and consists of a partial transformation of the second abdominal segment (A2) towards A1. The strongest phenotype appears in hemizygous combinations or in combinations with some of the lethal $a b d-A$ alleles described above. Of the $a b d-A$ domain, only the anterior compartment of A2 is affected by the mutation; the bristles of the dorsal side (tergite) become smaller and finer, resembling those of A1 (Fig. 1B). The number of bristles on the ventral side (sternite) is reduced as the A2 sternite is transformed towards the A1, which has no bristles. For example, in the $\mathrm{A} 2$ sternite, there are $15 \pm 2$ bristles in the wild type and $2 \pm 1$ in $i a b-2^{\mathrm{K}} / a b d-A^{\mathrm{M} 1}$. In addition the Wheeler's organ, a characteristic structure of $\mathrm{A} 2$, is nearly always absent. The homozygous $i a b-2^{\mathrm{K}}$ phenotype is similar but weaker.

However, we observed that $i a b-2^{\mathrm{K}} /+$ and $i a b-2^{\mathrm{K}} /$ $i a b-2^{\mathrm{K}}$ flies frequently present in the $\mathrm{A} 1$ tergite some bristles that are longer and thicker than those of $\mathrm{A} 1$, suggesting a partial transformation of A1 towards A2 (or other more posterior tergite). Since the A2 pattern is established by $a b d-A$ activity, which is not normally active in $\mathrm{A} 1$ (part of the $U b x$ domain), this observation suggests that in $i a b-2^{\mathrm{K}}$, together with a loss of function in A2, there is some ectopic activity of $a b d-A$ in A1.

This gain-of-function phenotype of $i a b-2^{\mathrm{K}}$ flies is dramatically enhanced if they are also made homozygous for $s u(H w)^{2}$, which suppresses most of the mutations caused by gypsy transposons (Modolell $e t$ al. 1983). In the doubly mutant flies, the tergite of $A 2$ is entirely normal but that of $\mathrm{A} 1$ is strongly transformed towards a more posterior segment (Fig. 1C). This transformation affects preferentially the tergite. In addition, we observed the presence of abdominal tissue anterior to A1. We have tested the segmental provenance of this tissue by generating en- $\beta$ gal $/+; s u(H w)^{2}$ $i a b-2^{\mathrm{K}} / s u(H w)^{2} i a b-2^{\mathrm{K}}$ flies in which the adult posterior compartments can be labelled by $\beta$-gal activity (Busturia and Morata, 1988). We find that the abdominal tissue anterior to $\mathrm{A} 1$ is located anterior to the T3p stripe and therefore is originated in the anterior compartment of the third thoracic segment.

Flies homozygous for $s u(H w)^{2}$ but heterozygous for $i a b-2^{\mathrm{K}}$ also show a clear, though milder, transformation of A1 (Fig. 1D), indicating that in the absence of the $s u(H w)$ product, $i a b-2^{\mathrm{K}}$ behaves as a dominant, gain-offunction mutation. Flies homozygous for $s u(H w)^{2}$ alone do not show this effect. All the genotypes generated for the study of $i a b-2^{\mathrm{K}}$ and two other $i a b-2$ mutations are presented in Table 2 . These observations indicate that the recessive (A2 towards A1) transformation of $i a b-2^{\mathrm{K}}$ flies is mostly or entirely due to the activity of the gypsy transposon, which requires the normal products of $s u(H w)$ (Parkhust and Corces, 1986; Mazo et al. 1989). 
A

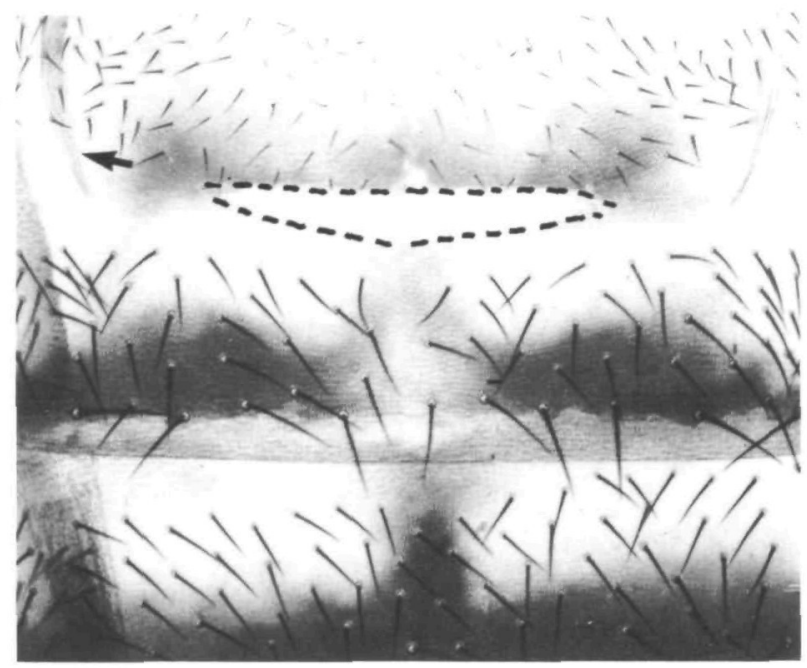

C

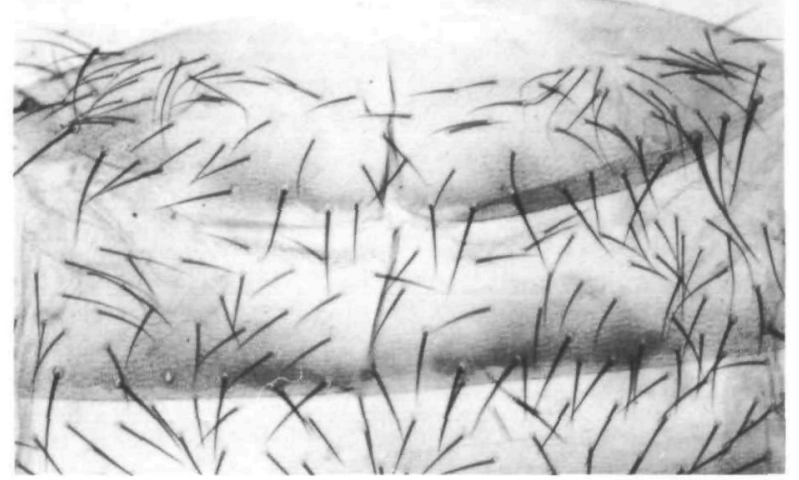

E

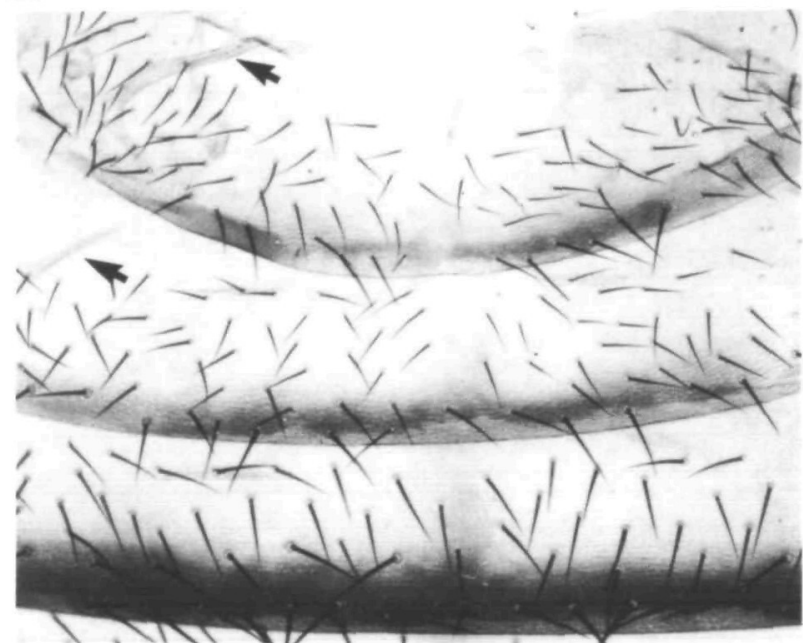

This gypsy activity probably interferes with the $a b d-A$ transcription unit in A2. Once the gypsy interference is removed by eliminating the $s u(H w)^{+}$, the $i a b-2^{\mathrm{K}}$
B

A1

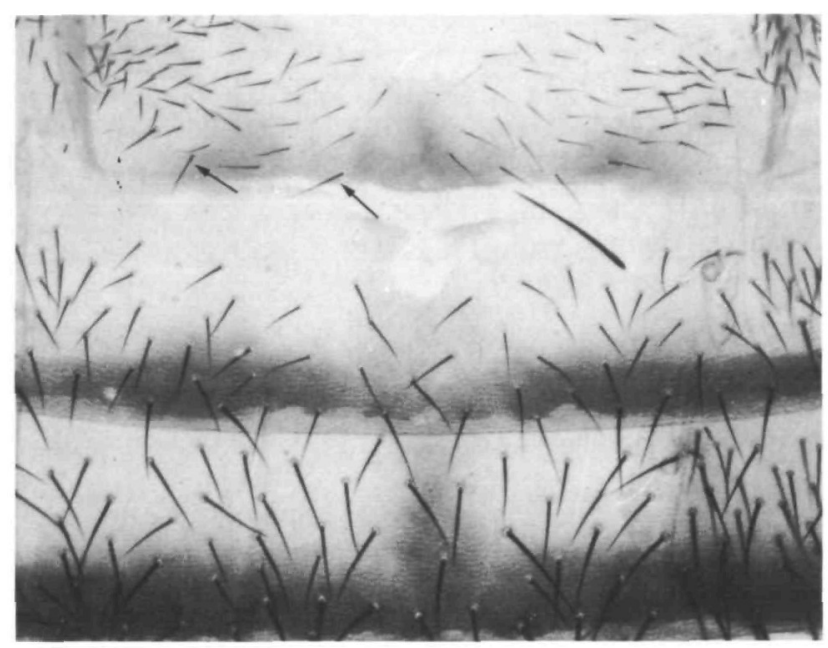

D

A1

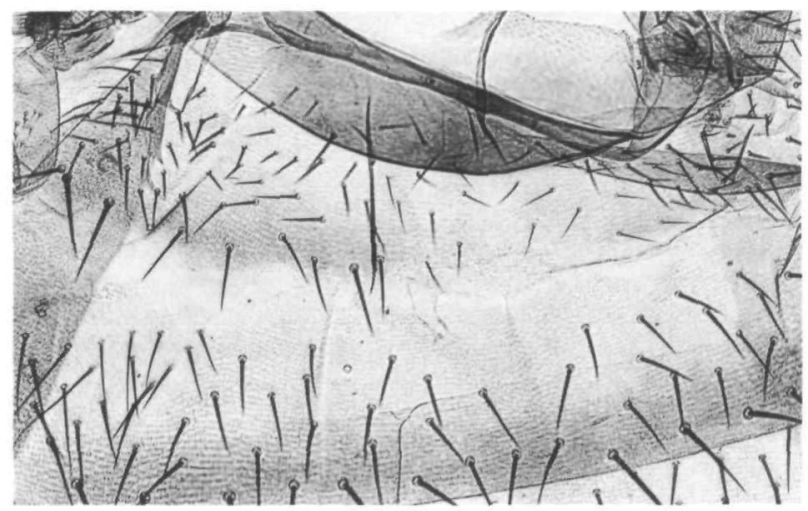

$\mathrm{F}$

A1

A2

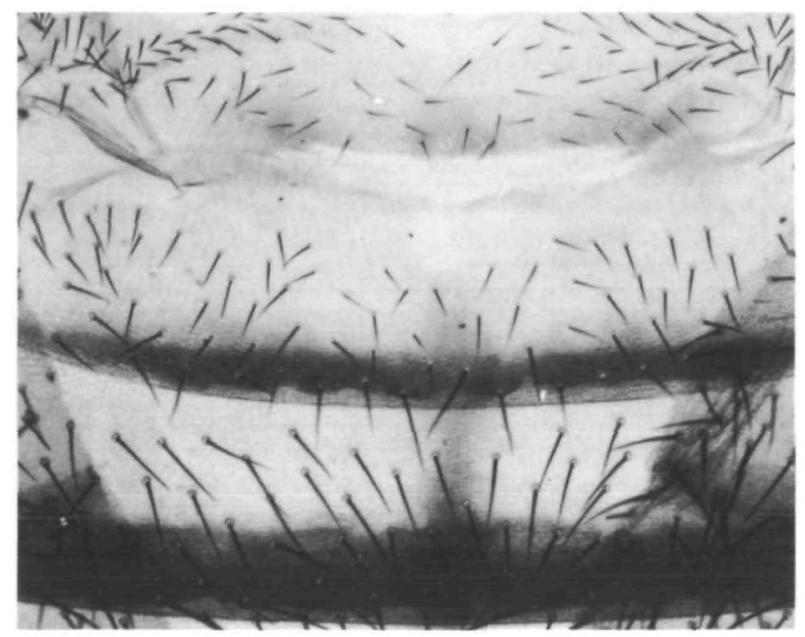

breakpoint reveals its genuine, dominant gain-of-function phenotype.

We have studied the embryonic phenotypes of $i a b$ - 
Fig. 1. Dorsal aspect of the first three abdominal segments in mutants affecting $\mathrm{A} 1$ and $\mathrm{A} 2$ development. (A) WT. Note that the bristles in $\mathrm{A} 1$ are shorter and finer than those of $\mathrm{A} 2$ and $\mathrm{A} 3$. There is also a characteristic cuticular arch (arrow). The posterior compartment of A1 (Kornberg, 1981 ) is a depigmented region (dotted line) devoid of bristles and is connected smoothly with A2a. In contrast, the posterior compartments of all other abdominal segments are not normally visible as they form a fold located under the anterior compartment. (B) $i a b-2^{\mathrm{K}} / D f$. The $\mathrm{A} 2$ segment is partially transformed into Al as indicated by the small size of the bristles. The transformation appears to be compartmental as A1p and A2p are not affected. Note in Ala (arrows) some bristles are longer than normal indicating a slight transformation towards a more posterior segment. (C) $s u(H w)^{2} i a b-2^{\mathrm{K}} / s u(H w)^{2} i a b-2^{\mathrm{K}}$. Almost complete transformation of A1 into A2. The transformation affects $\mathrm{A} 1 \mathrm{a}$ as indicated by the size of the bristles and the disappearance of the cuticular arch. A1p is also transformed, generating a discontinuity between $\mathrm{A} 1$ and A2. (D) $s u(H w)^{2} i a b-2^{\mathrm{R}} / s u(H w)^{2}$. This transformation is similar to but weaker than that of C. Both A1a and A1p are affected. (E) $i a b-2^{\mathrm{Uab} 1} / a b d-A^{\mathrm{Ml}}$. Reciprocal partial transformation of $\mathrm{A} 1$ towards $\mathrm{A} 2$ and of $\mathrm{A} 2$ towards $\mathrm{A} 1$. As a result $A 1$ and $A 2$ look quite similar (compare with WT). The transformation affects both A1a and A1p. Note the presence of the characteristic cuticular arch (arrows) in the two segments. (F) $i a b-2^{\mathrm{M} 1} / D f$. Partial transformation of A2a towards A1a. The existence of the discontinuity between $\mathrm{A} 2$ and $\mathrm{A} 3$ suggests that $\mathrm{A} 2 \mathrm{p}$ is not transformed. Alp also remains normal.

$2^{\mathrm{K}} / D f$ and of $s u(H w)^{2} i a b-2^{\mathrm{K}} / s u(H w)^{2} i a b-2^{\mathrm{K}}$ and found in both cases a normal pattern in the larval epidermis. Thus the phenotypes associated with $i a b-2^{\mathrm{K}}$ appear to be restricted to imaginal cells.

\section{Infraabdominal-2 $2^{\mathrm{S} 3}\left(\mathrm{i} a b-2^{\mathrm{S} 3}\right)$}

It is associated with a breakpoint in the position $+26 \mathrm{~kb}$ (Karch et al. 1985), very close to that of the gypsy insert of $i a b-2^{\mathrm{K}}(+27.5 \mathrm{~kb})$. It also shows a transformation of $\mathrm{A} 2$ towards $\mathrm{A} 1$, but is weaker than that of $i a b-2^{\mathrm{K}}$. A mild transformation can be observed in hemizygous

Table 2. Transformations caused by iab-2 mutations in $A 1$ and $A 2$ segments

\begin{tabular}{|c|c|c|}
\hline Genotype & Segmen & dentity \\
\hline 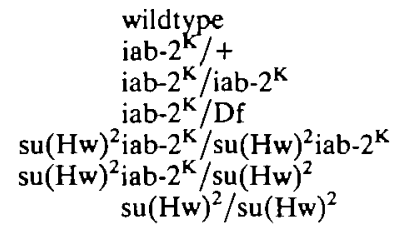 & $\begin{array}{l}\mathrm{A} 1 \\
\mathrm{~A} 1 \rightarrow \mathrm{A} 2(\mathrm{~s}) \\
\mathrm{A} 1 \rightarrow \mathrm{A} 2(\mathrm{~s}) \\
\mathrm{A} 1 \rightarrow \mathrm{A} 2(\mathrm{~s}) \\
\mathrm{A} 1 \rightarrow \mathrm{A} 2 \\
\mathrm{~A} 1 \rightarrow \mathrm{A} 2 \\
\mathrm{~A} 1\end{array}$ & $\begin{array}{l}\text { A2 } \\
\text { A2 } \\
\text { A2 } \rightarrow \text { A1 } \\
\text { A2 } \rightarrow \text { A1 } \\
\text { A2 } \\
\text { A2 } \\
\text { A2 }\end{array}$ \\
\hline 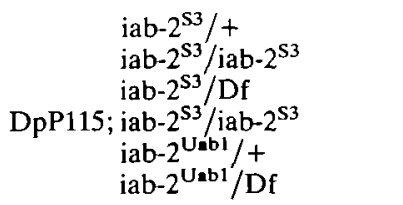 & $\begin{array}{l}\mathrm{A} 1 \rightarrow \mathrm{A} 2(\mathrm{~s}) \\
\mathrm{A} 1 \rightarrow \mathrm{A} 2(\mathrm{~s}) \\
\mathrm{A} 1 \rightarrow \mathrm{A} 2(\mathrm{~s}) \\
\mathrm{A} 1 \rightarrow \mathrm{A} 2(\mathrm{~s}) \\
\mathrm{A} 1 \rightarrow \mathrm{A} 2 \\
\mathrm{~A} 1 \rightarrow \mathrm{A} 2\end{array}$ & $\begin{array}{l}\mathrm{A} 2 \\
\mathrm{~A} 2 \rightarrow \mathrm{A} 1(\mathrm{~s}) \\
\mathrm{A} 2 \rightarrow \mathrm{A} 1 \\
\mathrm{~A} 2 \\
\mathrm{~A} 2 \\
\mathrm{~A} 2 \rightarrow \mathrm{A} 1\end{array}$ \\
\hline
\end{tabular}

(s) Indicates a slight transformation, usually only discernible under the compound microscope. flies as some reduction in the number of bristles in A2 sternite.

Given the proximity to the $i a b-2^{\mathrm{K}}$ lesion, we checked for a possible gain-of-function phenotype in $\mathrm{A} 1$ and found that indeed the A1 tergite shows some large bristles indicative of transformation towards a more posterior segment. This phenotype can be observed in heterozygous $i a b-2^{\mathrm{S} 3} /+$ flies but it is enhanced in homozygotes. It can also be observed in flies carrying supernumerary doses of the BX-C, indicating that the transformation of $\mathrm{A} 1$ is a genuine gain-of-function phenotype.

\section{Infraabdominal-2 $2^{\mathrm{Uab} 1}\left(\mathrm{iab}-2^{\mathrm{Uab} 1}\right)$}

This mutation shows a dominant and a recessive phenotype. Hemizygous $i a b-2^{\text {Uab1 }} / D f$ adults show partial transformations of A1 into A2 (described by Davis and Kiger, 1977 and Lewis, 1978) and of A2 into A1. As a result segments $\mathrm{A} 1$ and $\mathrm{A} 2$ look very much alike (Fig. 1E); other abdominal segments are not affected. The same phenotype appears in $i a b-2^{\mathrm{Uab} 1} / a b d-A^{\mathrm{M} 1}$. In $i a b-2^{\mathrm{Uab} 1} /+$ or DPP115; $i a b-2^{\mathrm{Uab} 1} / D f$ adults, which have one normal $a b d-A$ gene, the transformation of $\mathrm{A} 1$ remains unaltered whereas that of $\mathrm{A} 2$ is completely suppressed. The transformation of $\mathrm{A} 1$ is also unaffected by supernumerary copies of $a b d-A$.

All these observations indicate that $i a b-2^{\mathrm{Uab} 1}$ produces adventitious activity of $a b d-A$ in $\mathrm{A} 1$, responsible for the dominant phenotype, and a reduction of the normal $a b d-A$ function in A2.

The dominant transformation of $\mathrm{A} 1$ seen in adults is not detected in $i a b-2^{\mathrm{Uab} 1} /+$ larvae. Even homozygous $i a b-2^{\mathrm{Uab} 1}$ embryos containing an extra dose of the BX-C (necessary to cover the recessive $b x d, a b d-A$ and $A b d$ $B$ phenotypes) display a normal segment pattern.

\section{Infraabdominal-2 $2^{\mathrm{M} 1}\left(\right.$ iab-2 $\left.2^{\mathrm{M} 1}\right)$}

It appeared spontaneously in our laboratory. In hemizygous adults, the A2 segment is transformed towards A1 with an expressivity similar to that of $i a b-2^{\mathrm{K}} / D f$ (Fig. 1F). However, unlike $i a b-2^{\mathrm{K}}$, it does not have any effect on the A1 segment.

Trans combinations with lethal $a b d-A$ alleles like $a b d-A^{\mathrm{M} 1}, a b d-A^{\mathrm{M} 2}, a b d-A^{\mathrm{D} 24}$ and $a b d-A^{\mathrm{C} 51}$ present similar phenotypes. There is no effect on A3 or any other segment, thus this allele has the features of a genuine $i a b-2$ mutation of the type predicted by Lewis, 1978 .

We have examined the embryonic phenotype in hemizygous condition and found it like the wild type.

\section{Infraabdominal $-3^{277}\left(i a b-3^{277}\right)$}

This mutation has been described by Karch et al. 1985 . It is an X-ray-induced partial revertant of $M c p$ and contains a breakpoint in $+64 \mathrm{~kb}$.

Adults of genotype $i a b-3^{2 \pi} / D f$ present a partial transformation of A1 towards A2 and of A3-A5 towards $\mathrm{A} 2$. These transformations are visualized by the appearance of Wheeler's organs, more or less defective, in all these segments. Although transformations of posterior compartments of abdominal segments are normally difficult to assess given the lack of 
A

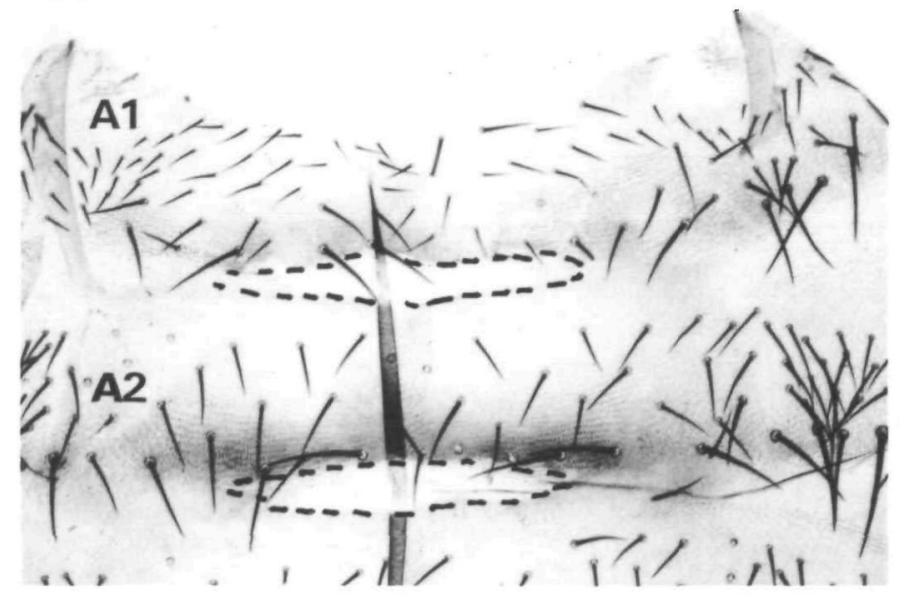

B

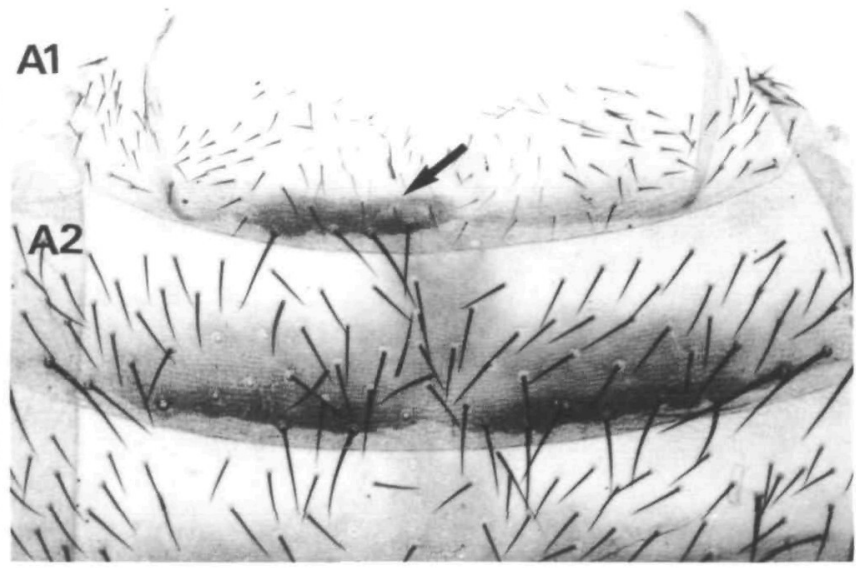

Fig. 2. Tergites of $\mathrm{A} 1$ and $\mathrm{A} 2$ in flies carrying $i a b$ mutations mapping $5^{\prime}$ to the transcription unit. (A) $i a b-3^{2 \pi} / D f$. A1a is partially transformed into A2a as suggested by the presence of large bristles (compare with WT in Fig. 1A). A1p remains unaltered but $\mathrm{A} 2 \mathrm{p}$ is transformed into $\mathrm{A} 1 \mathrm{p}$. Both $\mathrm{A} 1 \mathrm{p}$ and $\mathrm{A} 2 \mathrm{p}$ are marked by dotted lines. (B) $i a b-4^{302} / i a b-4^{302}$. A partial transformation of A1a towards a more posterior segment is indicated by a group of long bristles (arrow). A1p is also transformed; the depigmented area smoothly continuous between A1 and A2 is replaced by cuticular folding characteristic of the posterior compartments of A2-A6 segments.

morphological landmarks, in good preparations we have observed that the posterior compartments of A2-A5 of these flies are transformed into A1p (Fig. 2A). Thus the anterior abdomen of $i a b-3^{277} / D f$ adults appear to be formed by A1p-A2a units, corresponding to parasegment 7 . The same set of transformations is observed in $i a b-3^{2 \pi} / a b d-A^{\mathrm{M} 1}$ and in homozygous $i a b-3^{277}$ adult flies. The $i a b-3^{277}$ mutation is therefore associated with a gain of function of $a b d-A$ activity in $\mathrm{A} 1$ and a relative loss in A3-A5. We have discriminated these two phenotypic components by generating adults with one or more copies of the normal $a b d-A$ gene; the genotypes $i a b-3^{277} /+$, Dp 115; iab$3^{277} /+$ and $D p P 115 ; i a b-3^{277} / i a b-3^{277}$ show suppression of the transformations of $\mathrm{A} 3-\mathrm{A} 5$ but $\mathrm{A} 1$ remains partially transformed towards A2.

\section{Infraabdominal-3 $3^{\mathrm{Uab} 4}\left(\mathrm{iab}-3^{\mathrm{Uab} 4}\right)$}

This mutation maps at +63 (Karch et al. 1985) and also has a dominant (Lewis, 1978) and a recessive phenotype. $i a b-3^{\text {Uab4 }} / D f$ adults show a phenotype similar to that of $i a b-3^{277} / D f ; \mathrm{A} 1$ is transformed towards $\mathrm{A} 2$ and A3-A5 become like A2. The posterior compartments are also transformed into A1p, although not as clearly as in $i a b-3^{277} / D f$. The transformation of $\mathrm{A} 1$ is stronger than in $i a b-3^{27}$ and in addition there is frequently a group of abdominal-like bristles located anterior to A1. By homology with the $s u /(H w)^{2} i a b-2^{\mathrm{K}} / s u(H w)^{2} i a b-2^{\mathrm{K}}$ phenotype, we interpret these bristles as originated in $\mathrm{T} 3 \mathrm{a}$, thus suggesting that the $i a b-3^{\mathrm{Uab} 4}$ phenotype extends to the thorax. We have distinguished the dominant and recessive phenotypic components by adding extra copies of $a b d-A . D p P 115 ; i a b-3^{\text {Uab } 4} / D f$, $i a b-3^{\mathrm{Uab} 4} /+$ and $D p P 115 ; i a b-3^{\text {Uab4 }} /+$ have A3-A5 segments wild type but the transformations of T3 and A1 remain as in $i a b-3^{\text {Uab4 }} / D f$.

\section{Infraabdominal-4 $4^{302}\left(\mathrm{iab}-4^{302}\right)$}

Karch et al. (1985), described the hemizygous phenotype and also the molecular lesion, a breakpoint at $+86 \mathrm{~kb}$. The segment A4 is transformed into A3 and A2 is transformed A3 as revealed by the loss or abnormal development of the Wheeler's organ and the alteration of the bristle pattern of the A2 sternite. We have confirmed their observations for $i a b-4^{302} / D f$ adults although the pigmentation pattern of A4 is variable and its transformation sometimes difficult to assess. We find the same phenotype in $i a b-4^{302} / a b d-A^{\mathrm{M} 1}$ and in $i a b$ $4^{302} / a b d-A^{\mathrm{M} 2}$ flies. Homozygous $i a b-4^{302}$ adults also show a similar phenotype but, in addition, the A1 segment, particularly the posterior compartment, is transformed towards a more posterior segment. The same phenotype is seen in A1 of $D p P 115 ; i a b-4^{302} / i a b$ $4^{302}$ flies (Fig. 2B).

\section{Infraabdominal-4 ${ }^{\mathrm{MX} 4}\left(\right.$ iab-4 $\left.{ }^{\mathrm{MX} 4}\right)$}

It has recently been $\mathrm{X}$-ray induced in our laboratory. It has some features in common with $i a b-4^{302}$ so we have labelled it as $i a b-4$. $i a b-4^{\mathrm{MX}} / D f$ adults show a transformation of A1, especially Alp, towards a more posterior segment. The sternite of A2 frequently has the Wheeler's organ abnormal or absent. The pigment pattern of the A4 tergite is modified and resembles A3. The hemitergites do not fuse well and leave a cleft in the medial region.

The effects seen in $\mathrm{A} 1$ and $\mathrm{A} 2$ are not recessive as they appear in heterozygous $i a b-4^{\mathrm{MX} 4} /+$ and in $D p 115$; $i a b-4^{\mathrm{Mx}} / D f$ adults. This indicates that $i a b-4^{\mathrm{MX} 4}$ produces some gain of function of $a b d-A$ in $\mathrm{A} 1$ and $\mathrm{A} 2$, together with a relative loss in A4. Unfortunately, the $a b d-A^{\mathrm{MX} 4}$ chromosome is homozygous lethal, most probably due to a second site mutation, so we did not 
study the effect of two doses of the gain-of-function phenotype of the mutation.

$$
\text { Infraabdominal-4, } 5^{\mathrm{DB}}\left(\text { iab-4, } 5^{\mathrm{DB}}\right)
$$

This mutation is caused by a DNA deletion from +83 to +113 (Karch et al. 1985). The deletion extends into $A b d-B$ as it removes a piece of DNA where several $A b d-B$ mutations map (Karch et al. 1985). However, the tester abd-A mutant chromosomes we have used contain $A b d-B^{+}$so the $A b d-B$ phenotypical component should have been eliminated.

Flies of the genotype $i a b-4,5^{\mathrm{DB}} / D$ for $i a b-4,5^{\mathrm{DB}} / a b d$ $A^{\mathrm{M} 1}$ show A1 slightly modified towards a more posterior segment; also the Wheeler's organ is sometimes abnormal suggesting a transformation of A2. In males, the A5 tergite is depigmented resembling that of A4. However, ia $b-4,5^{\mathrm{DB}} /+$ males also present a partially depigmented A5 tergite suggesting that the A5 depigmentation may be in part due the haplo insufficiency of the $A b d-B$ fragment missing in the $i a b-4,5^{\mathrm{DB}}$ chromosome. The transformations in A1 and A2 are stronger in homozygous flies but are not affected by supernumerary doses of $a b d-A^{+}$indicating that the effects seen in $\mathrm{A} 1$ and $\mathrm{A} 2$ are due to gain of function of $a b d-A$.

\section{(4) Complementation studies}

The $a b d-A$ locus is defined as a single complementation group (Sánchez-Herrero et al. 1985). The abd- $A$ mutations do not complement among themselves and also fail to complement the $i a b$ alleles.

Complementation analyses among the $i a b$ alleles are complicated by the fact that, as we show above, many of them exhibit a combination of gain- and loss-of-function phenotypes. For complementation we have only considered the latter.

Trans-heterozygous combinations of $i a b-2$ alleles show the characteristic transformation of A2 towards $\mathrm{A} 1$, except in $i a b-2^{\mathrm{S} 3} / i a b-2^{\mathrm{Uab} 1}$ flies that show no detectable effect in A2 (A1 is strongly transformed to A2).

The combinations of $i a b-2$ with $i a b-3$ alleles show full complementation for the recessive phenotypes of both classes. Similarly trans combinations of $i a b-2$ and $i a b-4$ have no recessive phenotype. The combination of the two $i a b-3$ alleles, $i a b-3^{277} / i a b-3^{\text {Uab4 }}$ produces the same mutant phenotype as homozygous $i a b-3^{277}$ flies. We find it very difficult to decide on the complementation of $i a b-3$ and $i a b-4$ alleles given the variability of the recessive phenotype but there is no transformation in the A3 segment, so they seem to complement as to this phenotype.

The trans combinations of $i a b$ alleles often produce a strong enhancement of the gain-of-function phenotypes in A1 and A2 that many of them have. This is particularly clear in combinations involving $i a b-2^{\mathrm{K}}$, $i a b-2^{\mathrm{S} 3}, i a b-3^{277}, i a b-4^{302}$ and $i a b-4^{\mathrm{MX} 4}$; For example, $100 \%$ of the $i a b-2^{\mathrm{S} 3} / i a b-3^{277}$ flies show transformation of $A 1$ into $A 2$ and a similar result is found for $i a b-2^{\mathrm{K}} / i a b-3^{277}$. In the genotype $i a b-4^{303} / i a b-4^{\mathrm{MX}}$, Ala is transformed in about $50 \%$ of the cases and Alp in the $100 \%$. The Wheeler's organ of A2 is always absent.

\section{Discussion}

Mutations altering the normal function of the $a b d-A$ gene can be classified into two broad categories. (1) Those that affect the whole $a b d-A$ domain producing a general loss of $a b d$-A function. We designate them as $a b d-A$ mutations. (2) Mutations that affect only part of the $a b d-A$ domain and presumably modify the spatial distribution of $a b d-A$ function. These are viable and we name them $i a b$ mutations.

The $a b d-A$ mutations are nearly always lethal, but we have detected phenotypic differences among them. There is a group formed by $a b d-A^{\mathrm{M} 1}, a b d-A^{\mathrm{M} 2}$, $a b d-A^{\mathrm{D} 24}$ and $a b d-A^{\mathrm{C} 51}$ whose phenotypes are indistinguishable from that of the deletion of the gene; both in their embryonic phenotypes and in the adult phenotypes of their combinations with $i a b$ alleles. They therefore eliminate or severely inactivate $a b d-A$ gene products.

A second group is composed of the mutations $a b d-A^{\mathrm{P} 10}, a b d-A^{\mathrm{C} 26}, a b d-A^{\mathrm{M} 3}, a b d-A^{\mathrm{MX} 1}, a b d-A^{\mathrm{MX} 2}$ and $a b d-A^{\mathrm{MX} 3}$ that produce weaker segmental transformations either in embryonic or in adults. However, the whole $a b d-A$ domain is affected and probably this is, except in $a b d-A^{\mathrm{M} 3}$, incompatible with viability.

The localization of the $a b d-A$ mutations defines a region between $+33 \mathrm{~kb}$ and $+57 \mathrm{~kb}$ in the DNA map of Karch et al. (1985). This coincides extremely well with the region of the gene encoding the homeobox-containing transcription unit, which spans from $+33 \mathrm{~kb}$ to +56.5-57 kb (F. Karch, personal communication). Most probably $a b d-A$ mutations directly interfere with this unit. This in turn strongly suggests that all functions of the gene are mediated by the protein products encoded by it. If there are other transcription units, they should have a subsidiary (or no) role.

We note that four not-null $a b d-A$ alleles map at either one of the ends of the transcription unit; $a b d-A^{\mathrm{P} 10}$ and $a b d-A^{\mathrm{C} 26}$ around +33 , and $a b d-A^{\mathrm{MX} 1}$ and $a b d-A^{\mathrm{M} \times 2}$ at the interval $+55-58 \mathrm{~kb}$. It might be that in these cases the protein coding unit is not directly affected or, if it is, it may produce a form of truncated protein that still retains some function. Our results predict that these chromosomes permit some $a b d-A$ function that should be detectable once an anti- $a b d-A$ antibody is available.

The $i a b$ mutations are viable and can be subdivided into phenotypic classes, although the distinction is not always neat. They always fail to complement $a b d-A$ mutations indicating that the function(s) defective in $i a b$ alleles act in cis on the $a b d-A$ transcription unit, in the manner that for example $b x d$ acts in $c i s$ on $U b x$ transcription (Hogness et al. 1985).

It also seems that $i a b-3$ and $i a b-4$ mutations complement as to the recessive transformation of $\mathrm{A} 3$ into $\mathrm{A} 2$, although the $\mathrm{A} 4$ to $\mathrm{A} 3$ transformation may remain. Thus a minimum of two distinct functions are defined, one located $3^{\prime}$ and the other $5^{\prime}$ the transcription unit.

Which is the nature of the functions defined by $i a b$ alleles? In our view, they have a regulatory role connected with the distribution of $a b d-A$ products in the different abdominal metameres. This is based on 


\section{GENETIC STRUCTURE OF THE abd-A GENE}

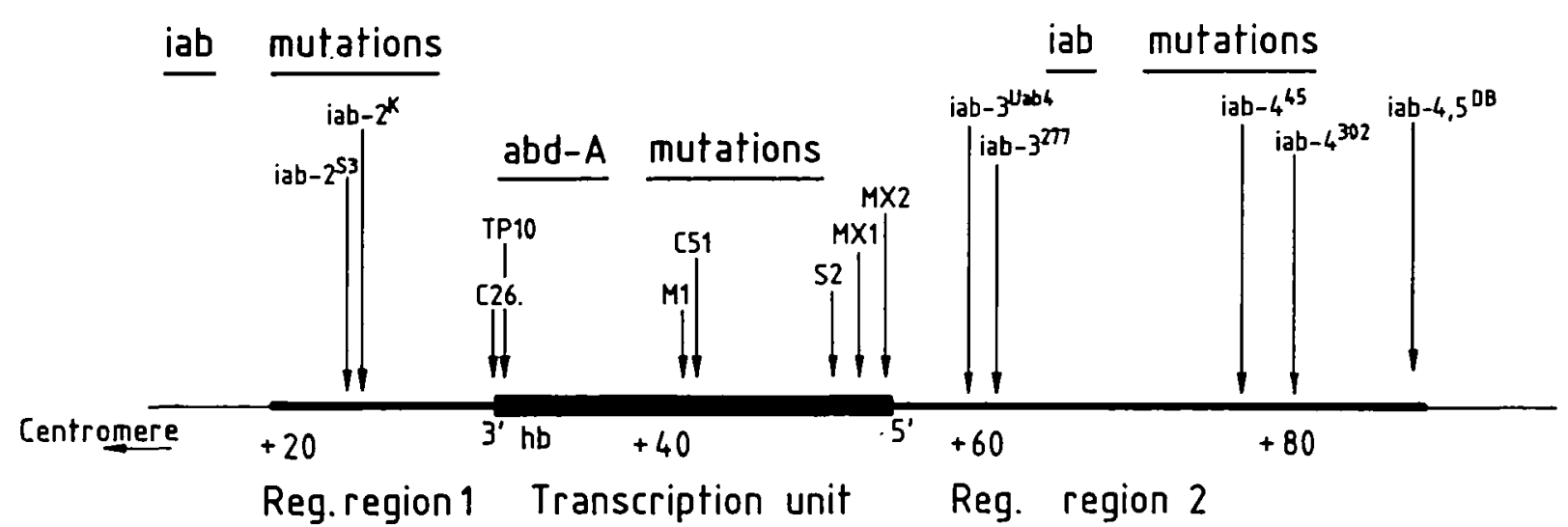

Ubx

abd-A

Abd-B

Fig. 3. Model of $a b d-A$ structure. The arrows point to the approximate positions of the lesions associated with the mutations regardless of whether they are breakpoints, inser-tions or deletions. The gene contains one transcription unit encoding all the executive products of the gene, and two regulatory regions connected with the spatial distribution of the gene products. Regulatory region 1 contains some control elements to prevent $a b d-A$ expression anterior to its normal domain and may also contain other elements to establish the appropriate level of $a b d-A$ product in A2. Regulatory region 2 appears to be connected with the general expression of $a b d-A$ in the abdomen since mutations in this region lead to a general alteration in the pattern of $a b d-A$ function (see text for details) affecting several segments. $h b$ indicates the approximate position of the homeobox. The positions of the mutations $a b d-A^{\mathrm{S} 2}$ and $i a b-4^{45}$, which have not been studied in this work, have been included as their phenotypes (Karch et al. 1985) match those of mutations in the same region.

two lines of argument. First, in $i a b$ mutants, part of the $a b d-A$ domain develops normally while other parts show loss of $a b d-A$ function, i.e. $a b d-2^{\mathrm{M} 1}$ transforms A2 but $\mathrm{A} 3$ and $\mathrm{A} 4$ are normal; in $i a b-3^{277}$, A2 is normal but $\mathrm{A} 3$ and $\mathrm{A} 4$ are transformed. This is expected if the distribution of $a b d-A$ products, rather that the function of the $a b d-A$ transcription unit is abnormal. The second argument is that many of these mutations exhibit a gainof-function phenotype in $\mathrm{A} 1$, outside the $a b d-A$ domain. This again suggests an abnormal distribution of $a b d-A$ products.

One important question is whether there exist within the $i a b$ regions individual elements responsible for $a b d-A$ expression in specific metameres, as proposed by recent models (Casanova et al. 1987; Peiferet al. 1987). i.e. whether there exist $i a b-2, i a b-3, i a b-4$ etc. as distinct genetic elements. The recessive phenotype of $i a b-2$ mutants suggests a metameric specific control (it is not clear whether compartmental, segmental or parasegmental). Also the observation that iab-4 mutations complement the recessive phenotype of $i a b-3$ alleles suggests that they affect distinct regulatory regions, perhaps metameric specific, at the 5 ' side of the gene. However, the $i a b-3$ mutations show in parasegments 8 and 9 a similar transformation to parasegment 7 . This would not be expected if there are distinct elements for parasegment $8(i a b-3)$ and $9(i a b-4)$. Besides we have shown that $i a b-3$ and $i a b-4$ mutations give rise to ectopic expression in A1 and A2 (or parasegments 6 and 7) and this is not easily compatible with models based on metameric specific regulatory elements. The simplest view about the $i a b-3$ and $i a b-4$ mutations is that they cause a general misregulation of $a b d-A$ expression. This results in an abnormal distribution of $a b d-A$ products, which now may appear inappropriately in the $U b x$ domain or at abnormally high levels in parts of the $a b d-A$ domain (Alp and A2a). This phenomenon would produce a gain-of-function, usually dominant, phenotype. Other parts of the $a b d-A$ domain (A2p, A3 and A4) would have a reduced level of product and consequently a loss-of-function phenotype. This interpretation has the advantage that offers a single explanation for a complex mutant syndrome.

One peculiar feature of many of these mutations is that the dominant transformations are restricted to adult structures; larval segments appear normal. This might be interpreted as indicating differential regulation for larval and adult cells. However, another possibility is that any dominant transformation affecting larval pattern may result in lethality. This phenomenon would result in the selection of mutations having little or no effect on larval patterns.

\section{A model of abd-A structure}

Our genetic results, together with the available information about the molecular mapping of $a b d-A \mathrm{mu}$ tations (Karch et al. 1985) suggest a model of $a b d-A$ structure outlined in Fig. 3. The model we propose is of 
necessity very general at this stage and will have to be refined in the light of subsequent molecular and genetic information.

All the protein products responsible for $a b d-A$ functions are encoded in the homeobox-containing transcription unit which is flanked by two cis-acting regulatory regions, 1 and 2 .

The transcription unit is responsible for all the executive products of the gene. Therefore mutations that impede its function present a null phenotype equivalent to the deletion of the entire gene. Little is still known about the different components of this unit, number, size and disposition of exons and introns etc.

Regulatory region 1 is located $3^{\prime}$ to the transcription unit and is defined by the $i a b-2^{\mathrm{K}}$ and $i a b-2^{\mathrm{S} 3}$ breakpoints. This part of the gene is connected with the repression of $a b d-A$ activity anterior to its domain, that is, it acts as a negative regulatory element. Another BX-C gene, $U b x$ appears to have similar components localized in a homologous position, defined by the mutations $C b x^{3}$ and $C b x^{\text {TWT }}$ (Bender et al. 1983).

However, both $i a b-2^{\mathrm{K}}$ (in the presence of $s u(H w)^{+}$) and $i a b-2^{\mathrm{S} 3}$ show a reduction of $a b d-A$ function in $\mathrm{A} 2$, suggesting they also interfere with another function necessary to promote $a b d-A$ activity in $\mathrm{A} 2$ and that should also be located $3^{\prime}$ to the transcription unit. The eventual localization of the DNA lesions of $i a b-2^{\mathrm{M} 1}$ and $i a b-2^{\text {Uab1 }}$ will help to further define these regions.

Regulatory region 2 extends for about $25 \mathrm{~kb}$ and is defined by the $i a b-3$ and $i a b-4$ mutations which provoke abnormal levels of $a b d-A$ function in A1-A4 segments. Unlike regulatory region 1 , whose role appears to be restricted to the $\mathrm{A} 2$ segment and part of the $U b x$ domain, the role of regulatory region 2 appears to be more general. It is connected with the general regulation of $a b d-A$ products in the abdomen. It is still an open question whether there are distinct components within it. Further genetic and molecular analysis of this region is still required.

We thank Peter Lawrence for comments in the manuscript and the Agencia Nacional de Evaluación and the Ramón Areces Foundation for financial suppport.

\section{References}

Bender, W., Akam, M., Karch, F., Beachy, P. A., Peifer, M., Spierer, P., Lewis, E. B. And Hogness, D. S. (1983). Molecular genetics of the bithorax complex in Drosophila melanogaster. Science 221, 23-29.

Busturla, A. and Morata, G. (1988). Ectopic expression of homeotic genes caused by the elimination of the Polycomb gene in Drosophila imaginal epidermis. Development 104, 713-720.

Casanova, J., Sánchez-Herrero, E., Busturia, A. and Morata, G. (1987). Double and triple mutant combinations of the bithorax complex of Drosophila. EMBO J. 6, 3103-3109.

Casanova, J., Sánchez-Herrero, E. and Morata, G. (1985a).

Prothoracic transformation and functional structure of the Ultrabithorax gene of Drosophila. Cell 42, 663-669.

Casanova, J., Sánchez-Herrero, E. and Morata, G. (1985b)

Contrabithorax and the control of spatial expression of the

bithorax complex genes of Drosophila. J. Embryol. exp. Morph. 90, 179-196.

Casanova, J., Sánchez-Herrero, E. and Morata, G. (1986).
Identification and characterization of a parasegment specific regulatory element of the Abdominal-B gene of Drosophila. Cell 47, 627-636.

Davis, R. C. AND Kiger, J. A. (1977). A clonal analysis of tergite development in Drosophila of Ultraabdominal and Paradoxical Genotypes. Devl Biol. 58, 114-123.

Duncan, I. (1987). The bithorax complex. A. Rev. Genet. 21, 285-319.

Hogness, D. S., Lipshitz, H. D. AND BeACHY, P. A. (1985) Regulation and products of the $U b x$ domain of the bithorax complex. Cold Spring Harbor Symp. Quant. Biol. 50, 181-194.

Karch, F., Weiffenbach, B., Peifer, M., Bender, W., Duncan, I., Celniker, S., Crosby, M. and Lewis, E. B. (1985). The abdominal region of the bithorax complex. Cell 43, 81-96.

Kornberg, T. (1981). Compartments in the abdomen of Drosophila and the role of the engrailed locus. Devl Biol. 86, 363-372.

Kuhn, D. T., Woods, D. F. And COOK, J. L. (1981). Analysis of a new homeotic mutation (iab-2) within the bithorax complex in Drosophila melanogaster. Mol. gen. Genet. 181, 82-86.

LewIs, E. B. (1978). A gene complex controlling segmentation in Drosophila. Nature, Lond. 275, 565-570.

Mazo, A. M., Mizrokhi, L. J., Karavanov, A. A., Sedkov, Y. A., Krichevskaja, A. A. ANd Ilyin, Y. V. (1989). Suppression in Drosophila: $s u(\mathrm{Hw})$ and $s u(f)$ gene products interact with a region of gypsy (mdg4) regulating its transcriptional activity. EMBO J. 8, 903-911.

McGinnis, W., Levine, M. S., Hafen, E., Kuroiwa, A. and GeHrING, W. (1984). A conserved DNA sequence in homeotic genes of Drosophila Antennapedia and bithorax complexes. Nature, Lond. 308, 428-433.

Mopolell, J., Bender, W. ANd Meselson, M. (1983). Drosophila melanogaster mutations suppressible by the Suppressor of Hairy wing are insertions of a 7,3 kilobase mobile element. Proc. natn. Acad. Sci. U.S.A. 80, 1678-1682.

Morata, G., Botas, J., Kerridge, S. and Struhl, G. (1983). Homeotic transformation of the abdominal segments of Drosophila caused by breaking or deleting a central portion of the bithorax complex. J. Embryol. exp. Morph. 78, 319-341.

Parkhust, S. M. And Corces, V. G. (1986). Interactions among the gypsy transposable element and the yellow and suppressor of Hairy wing loci in Drosophila melanogaster. Molec. cell. Biol. 6, 47-53.

Peifer, M. ANd Bender, W. (1986). The anterobithorax and bithorax mutations of the bithorax complex. EMBO J. 5, 2293-2303.

Peifer, M., Karch, F. And Bender, W. (1987). The bithorax complex: control of segment identity. Genes and Development 1 , $891-898$.

Regulski, M., Katherine, H., Kostriken, R., Karch, F., Levine, M. AND MCGinNis, W. (1985). Homeo box genes of the Antennapedia and Bithorax Complex of Drosophila. Cell 43, 71-80.

Rowe, A. And AKam, M. (1988). The structure and expression of a hybrid homeotic gene. EMBO J. 7, 1107-1114.

Sánchez-Herrero, E., Vernos, I., Marco, R. and Morata, G. (1985). Genetic organization of the Drosophila Bithorax complex. Nature, Lond. 313, 108-113.

Scott, M. P. AND Weiner, A. J. (1984). Structural relationships among genes that control development: sequence homology between the Antennapedia, Ultrabithorax and fushi tarazu loci of Drosophila. Proc. natn. Acad. Sci., U.S.A. 81, 4115-4119.

Tiong, S., Bone, L. M. and Whittle, J. R. S. (1985). Recessive lethal mutations within the bithorax complex of Drosophila. Molec. gen. Genet. 200, 335-342.

VAN DER MeER, J. M. (1977). Optically clean and permanent whole mount preparation for phase constrast microscopy of cuticular structures of insect larvae. Drosophila Information Service 52, 160 . 